\title{
Random Eigenvalue Problems in Structural Dynamics
}

\author{
S. Adhiakri* and M. I. Friswell \\ University of Bristol, Bristol, United Kingdom
}

\begin{abstract}
Dynamic characteristics of linear structural systems are governed by the natural frequencies and the mode-shapes. In this paper moments and probability density functions of the eigenvalues of linear stochastic dynamic systems are considered. It is assumed that the mass and the stiffness matrices are smooth and at least twice differentiable functions of a random parameter vector. The random parameter vector is assumed to be non-Gaussian in general. Current methods to solve such problems are dominated by perturbation based methods. Here a new approach based on an asymptotic approximation of the multidimensional integrals is proposed. A closedform expression is derived for a general $r$ th order moment of the eigenvalues. Two approaches are presented to obtain the probability density functions of the eigenvalues. The first is based on the maximum entropy method and the second is based on fitting of a chi-square random variable. Both approaches result in simple closedform expressions which can be easily calculated. The proposed methods are applied to a three degrees-of-freedom spring-mass system and the results are compared with Monte Carlo simulations. Two different cases, namely (a) when all eigenvalues are well separated, and (b) when some eigenvalues are closely spaced, are considered to illustrate some inherent properties of the methodologies proposed in the paper.
\end{abstract}

\section{Nomenclature}

\begin{tabular}{ll}
$\mathbf{D}_{(\bullet)}(\mathbf{x})$ & Hessian matrix of $(\bullet)$ at $\mathbf{x}$ \\
$\mathbf{d}_{(\bullet)}(\mathbf{x})$ & gradient vector of $(\bullet)$ at $\mathbf{x}$ \\
$\mathbf{I}$ & identity matrix \\
$\mathbf{K}$ & stiffness matrix \\
$\mathbf{M}$ & mass matrix \\
$\mathbf{x}$ & basic random variables \\
$L(\mathbf{x})$ & negative of the log-likelihood function \\
$m$ & number of basic random variables \\
$N$ & degrees-of-freedom of the system \\
$n$ & number of moments used for pdf construction \\
$p_{(\bullet)}$ & probability density function of $(\bullet)$ \\
$\boldsymbol{\alpha}$ & optimal point for perturbation method \\
$\boldsymbol{\mu}$ & mean of parameter vector $\mathbf{x}$ \\
$\boldsymbol{\phi}_{j}$ & eigenvectors of the system \\
$\boldsymbol{\Sigma}$ & covariance matrix \\
$\boldsymbol{\theta}$ & optimal point for asymptotic method \\
$\mathcal{L}$ & Lagrangian \\
$\epsilon_{m}, \epsilon_{k}$ & strength parameters associated with mass and stiffness coefficients \\
$\eta_{j}, \gamma_{j}, \nu_{j}$ & parameters of $\chi^{2}$-approximated pdf of $\lambda_{j}$ \\
$\lambda_{j}$ & eigenvalues of the system \\
$\mu_{j}^{(r)}$ & rth order moment of the eigenvalues \\
\hline$(\bullet)$ & deterministic value of $(\bullet)$ \\
$\Phi$ & cumulative Gaussian distribution function \\
$\rho_{r}$ & Lagrange multipliers, $r=0,1,2, \cdots n$ \\
&
\end{tabular}

${ }^{*}$ Corresponding author: Department of Aerospace Engineering, Queens Building, University Walk, Bristol BS8 1TR, UK 


$\begin{array}{ll}\sigma_{j} & \text { standard deviation of } \lambda_{j} \\ (\bullet)^{T} & \text { matrix transpose } \\ \approx & \text { approximately equal to } \\ \mathbb{R} & \text { space of real numbers } \\ \|\bullet\| & \text { determinant of matrix }(\bullet) \\ \mathrm{E}[\bullet] & \text { mathematical expectation operator } \\ \in & \text { belongs to } \\ \longmapsto & \text { maps into } \\ \widehat{(\bullet)} & \text { mean of }(\bullet) \\ \text { dof } & \text { degrees-of-freedom } \\ \text { pdf } & \text { probability density function }\end{array}$

\section{Introduction}

Characterization of the natural frequencies and mode-shapes play a fundamental role in the analysis and design of engineering dynamic systems. The determination of natural frequency and mode shapes require the solution of an eigenvalue problem. Eigenvalue problems also arise in the context of the stability analysis of structures. This problem could either be a differential eigenvalue problem or a matrix eigenvalue problem, depending on whether a continuous model or a discrete model is used to describe the given vibrating system. Description of real-life engineering structural systems is inevitably associated with some amount of uncertainty in specifying material properties, geometric parameters, boundary conditions and applied loads. For these reasons it is necessary to consider random eigenvalue problems. Several studies have been conducted on this topic since the mid-sixties. The study of probabilistic characterization of the eigensolutions of random matrix and differential operators is now an important research topic in the

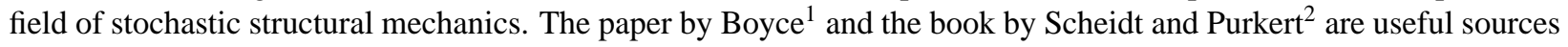
of information on early work in this area of research and also provide a systematic account of different approaches to random eigenvalue problems. Several review papers $3,4,5,6,7$ have appeared in this field which summarize the current as well as the earlier works.

In this paper discrete linear systems or discretized continuous systems are considered. The random eigenvalue problem of undamped or proportionally damped systems can be expressed by

$$
\mathbf{K}(\mathbf{x}) \phi_{j}=\lambda_{j} \mathbf{M}(\mathbf{x}) \phi_{j} .
$$

Here $\lambda_{j}$ and $\phi_{j}$ are the eigenvalues and the eigenvectors of the dynamic system. $\mathbf{M}(\mathbf{x}) \in \mathbb{R}^{N \times N}$ and $\mathbf{K}(\mathbf{x}) \in \mathbb{R}^{N \times N}$, the mass and stiffness matrices, are assumed to be smooth, continuous and at least twice differentiable functions of a random parameter vector $\mathbf{x} \in \mathbb{R}^{m}$. The vector $\mathbf{x}$ may consist of material properties, e.g., mass density, Poisson's ratio, Young's modulus; geometric properties, e.g., length, thickness, and boundary conditions. Statistical properties of the system are completely described by the joint probability density function $p_{\mathbf{x}}(\mathbf{x}): \mathbb{R}^{m} \mapsto \mathbb{R}$. For mathematical convenience we express

$$
p_{\mathbf{X}}(\mathbf{x})=e^{-L(\mathbf{x})}
$$

where $-L(\mathbf{x})$ is often known as the log-likelihood function. For example, if $\mathbf{x}$ is a $m$-dimensional multivariate Gaussian random vector with mean $\boldsymbol{\mu} \in \mathbb{R}^{m}$ and covariance matrix $\boldsymbol{\Sigma} \in \mathbb{R}^{m \times m}$ then

$$
L(\mathbf{x})=\frac{m}{2} \ln (2 \pi)+\frac{1}{2} \ln \|\boldsymbol{\Sigma}\|+\frac{1}{2}(\mathbf{x}-\boldsymbol{\mu})^{T} \boldsymbol{\Sigma}^{-1}(\mathbf{x}-\boldsymbol{\mu})
$$

In this paper it is assumed that in general the random parameters are non-Gaussian and correlated, i.e., $L(\mathbf{x})$ can have any general form provided it is a smooth, continuous and at least twice differentiable function. It is further assumed that $\mathbf{M}$ and $\mathbf{K}$ are symmetric and positive definite random matrices so that all the eigenvalues are real and positive.

The central aim of studying random eigenvalue problems is to obtain the joint probability density function of the eigenvalues and the eigenvectors. Under very special circumstances when the matrix $\mathbf{M}^{-1} \mathbf{K}$ is GUE (Gaussian unitary ensemble) or GOE (Gaussian orthogonal ensemble) an exact closed-form expression can be obtained for the joint pdf of the eigenvalues. ${ }^{8}$ In general the system matrices for real structures are not GUE or GOE and consequently some kind of approximate analysis is required. The current literature on random eigenvalue problems arising in engineering systems is dominated by the mean-centered perturbation methods. These methods work well when the uncertainties are small and the parameter distribution is Gaussian. In theory, any set of non-Gaussian random variables can be transformed into a set of standard Gaussian random variables by using numerical transformations such as the Rosenblatt transformation or the Nataf transformation. These transformations are often complicated, numerically expensive and inconvenient to implement in practice. 
In this paper a new approach is presented to obtain the moments and probability density functions of the eigenvalues. The proposed approach does not require the Gaussian pdf assumption of the basic random variables often employed in literature. Moreover, it also eliminates the need to use intermediate numerical transformations of the basic random variables. In Section II perturbation based methods are reviewed in the context of non-Gaussian basic random variables. In Section III a new method to obtain arbitrary order moments of the eigenvalues is proposed. Using these moments, some closed-form expressions of approximate pdf of the eigenvalues are derived in Section IV. In Section V the proposed analytical methods are applied to a three-dof problem and the results are compared to Monte Carlo simulation.

\section{Review of Perturbation Based Methods}

\section{A. Mean-Centered Perturbation Method}

The mass and the stiffness matrices are in general non-linear functions of the random vector $\mathbf{x}$. Denote the mean of $\mathbf{x}$ as $\boldsymbol{\mu} \in \mathbb{R}$, and consider that

$$
\mathbf{M}(\boldsymbol{\mu})=\overline{\mathbf{M}}, \quad \text { and } \quad \mathbf{K}(\boldsymbol{\mu})=\overline{\mathbf{K}}
$$

are the 'deterministic parts' of the mass and stiffness matrices respectively. In general $\overline{\mathbf{M}}$ and $\overline{\mathbf{K}}$ are different from the mean matrices. The deterministic part of the eigenvalues, $\lambda_{j}(\boldsymbol{\mu})=\bar{\lambda}_{j}$ is obtained from the deterministic eigenvalue problem $\overline{\mathbf{K}} \bar{\phi}_{j}=\bar{\lambda}_{j} \overline{\mathbf{M}} \bar{\phi}_{j}$.

The eigenvalues, $\lambda_{j}(\mathbf{x}): \mathbb{R}^{m} \mapsto \mathbb{R}$ are non-linear functions of the parameter vector $\mathbf{x}$. If the eigenvalues are not repeated, then each $\lambda_{j}(\mathbf{x})$ is expected to be a smooth and twice differentiable function since the mass and stiffness matrices are smooth and twice differentiable functions of the random parameter vector. In the mean-centered perturbation approach the function $\lambda_{j}(\mathbf{x})$ is expanded by its Taylor series about the point $\mathbf{x}=\boldsymbol{\mu}$ as

$$
\lambda_{j}(\mathbf{x}) \approx \lambda_{j}(\boldsymbol{\mu})+\mathbf{d}_{\lambda_{j}}^{T}(\boldsymbol{\mu})(\mathbf{x}-\boldsymbol{\mu})+\frac{1}{2}(\mathbf{x}-\boldsymbol{\mu})^{T} \mathbf{D}_{\lambda_{j}}(\boldsymbol{\mu})(\mathbf{x}-\boldsymbol{\mu})
$$

Here $\mathbf{d}_{\lambda_{j}}(\boldsymbol{\mu}) \in \mathbb{R}^{m}$ and $\mathbf{D}_{\lambda_{j}}(\boldsymbol{\mu}) \in \mathbb{R}^{m \times m}$ are respectively the gradient vector and the Hessian matrix of $\lambda_{j}(\mathbf{x})$ evaluated at $\mathbf{x}=\boldsymbol{\mu}$, that is

$$
\begin{aligned}
\left\{\mathbf{d}_{\lambda_{j}}(\boldsymbol{\mu})\right\}_{k} & =\left.\frac{\partial \lambda_{j}(\mathbf{x})}{\partial x_{k}}\right|_{\mathbf{x}=\boldsymbol{\mu}} \\
\text { and } \quad\left\{\mathbf{D}_{\lambda_{j}}(\boldsymbol{\mu})\right\}_{k l} & =\left.\frac{\partial^{2} \lambda_{j}(\mathbf{x})}{\partial x_{k} \partial x_{l}}\right|_{\mathbf{x}=\boldsymbol{\mu}} .
\end{aligned}
$$

Expressions of the elements of the gradient vector and the Hessian matrix are given in Appendix A. Equation (5) implies that the eigenvalues are effectively expanded about their corresponding deterministic value $\bar{\lambda}_{j}$.

Equation (5) represents a quadratic form in basic non-Gaussian random variables. The first-order perturbation, which is often used in practice, is obtained from Eq. (5) by neglecting the Hessian matrix. In this case the eigenvalues are simple linear functions of the basic random variables. This formulation is expected to produce acceptable results when the random variation in $\mathbf{x}$ is small. If the basic random variables are Gaussian then first-order perturbation results in a Gaussian distribution of the eigenvalues. In this case a closed-form expression for their joint probability density function can be obtained easily, see for example, Collins and Thomson, ${ }^{9}$ Anantha Ramu and Ganesan ${ }^{10,11,12,13}$ and Sankar et al. ${ }^{14}$ More recently Adhikari ${ }^{15,16}$ used a first-order perturbation method for complex eigenvalue problems arising in non-proportionally damped systems.

If the second-order terms are retained in Eq. (5) and the basic random variables are Gaussian, each $\lambda_{j}(\mathbf{x})$ results in a quadratic form in Gaussian random variables. Extensive discussions on such quadratic forms can be found in the books by Johnson and Kotz ${ }^{17}$ (Chapter 29) and Mathai and Provost. ${ }^{18}$ Using the methods outlined in these references it is possible to obtain moments, cumulants and the pdf of $\lambda_{j}(\mathbf{x})$. However, if the elements of $\mathbf{x}$ are non-Gaussian then the first-order perturbation or the second-order perturbation methods are not very helpful because there is no general method to obtain the resulting pdf in a simple manner.

\section{B. Perturbation Method Based on an Optimal Point}

In the mean-centered perturbation method, $\lambda_{j}(\mathbf{x})$ is expanded in a Taylor series about $\mathbf{x}=\boldsymbol{\mu}$. This approach may not be very suitable for all problems, especially if $\mathbf{x}$ is non-Gaussian then $p_{\mathbf{X}}(\mathbf{x})$ may not be centered around the mean. Recently Adhikari and Langley ${ }^{19}$ have proposed a method which searches for a point $\mathbf{x}=\boldsymbol{\alpha}$ in the $\mathbf{x}$-space such that the Taylor series expansion of $\lambda_{j}(\mathbf{x})$ about this point

$$
\lambda_{j}(\mathbf{x}) \approx \lambda_{j}(\boldsymbol{\alpha})+\mathbf{d}_{\lambda_{j}}^{T}(\boldsymbol{\alpha})(\mathbf{x}-\boldsymbol{\alpha})+\frac{1}{2}(\mathbf{x}-\boldsymbol{\alpha})^{T} \mathbf{D}_{\lambda_{j}}(\boldsymbol{\alpha})(\mathbf{x}-\boldsymbol{\alpha})
$$


is optimal in some sense. Here we extend the approach to the case when the basic random variables are non-Gaussian.

The optimal point $\boldsymbol{\alpha}$ can be selected in various ways. For practical applications the mean of the eigenvalues is often the most important. For this reason, the optimal point $\boldsymbol{\alpha}$ is selected such that the mean or the first moment of each eigenvalue is calculated most accurately. The mathematical formalism presented here is not restricted to this specific criteria and can be easily modified if any moment other than the first moment is required to be obtained more accurately. Using Eq. (2) the mean of $\lambda_{j}(\mathbf{x})$ can be obtained as

$$
\begin{aligned}
& \widehat{\lambda}_{j}=\mathrm{E}\left[\lambda_{j}(\mathbf{x})\right]=\int_{\mathbb{R}^{m}} \lambda_{j}(\mathbf{x}) p_{\mathbf{x}}(\mathbf{x}) d \mathbf{x}=\int_{\mathbb{R}^{m}} \lambda_{j}(\mathbf{x}) e^{-L(\mathbf{x})} d \mathbf{x}=\int_{\mathbb{R}^{m}} e^{-h_{j}(\mathbf{x})} d \mathbf{x} \\
& \text { where } \quad h_{j}(\mathbf{x})=L(\mathbf{x})-\ln \lambda_{j}(\mathbf{x}) .
\end{aligned}
$$

Evaluation of the integral (9), either analytically or numerically, is in general difficult because (a) $\lambda_{j}(\mathbf{x})$ and $L(\mathbf{x})$ are complicated nonlinear functions of $\mathbf{x}$, (b) an explicit functional form $\lambda_{j}(\mathbf{x})$ is not easy to obtain except for very simple problems (usually an FE run is required to obtain $\lambda_{j}$ for every $\mathbf{x}$ ), and (c) the dimension of the integral $m$ is large. For these reasons some kind of approximation is required. From Eq. (9) note that the maximum contribution to the integral comes from the neighborhood where $h_{j}(\mathbf{x})$ is minimum. Therefore, expand the function $h_{j}(\mathbf{x})$ in a Taylor series about a point where $h_{j}(\mathbf{x})$ has its global minimum. By doing so the error in evaluating the integral (9) would be minimized. Thus, the optimal point can be obtained from

$$
\frac{\partial h_{j}(\mathbf{x})}{\partial x_{k}}=0 \quad \text { or } \quad \frac{\partial L(\mathbf{x})}{\partial x_{k}}=\frac{1}{\lambda_{j}(\mathbf{x})} \frac{\partial \lambda_{j}(\mathbf{x})}{\partial x_{k}}, \quad \forall k .
$$

Combining the above equation for all $k$, at $\mathbf{x}=\boldsymbol{\alpha}$ we have

$$
\mathbf{d}_{\lambda_{j}}(\boldsymbol{\alpha})=\lambda_{j}(\boldsymbol{\alpha}) \mathbf{d}_{L}(\boldsymbol{\alpha})
$$

Equation (12) implies that at the optimal point the gradient vectors of the eigenvalues and log-likelihood function are parallel. The non-linear set of equations (12) have to be solved numerically. A good 'first guess' to start the numerical solution process is $\boldsymbol{\alpha}=\boldsymbol{\mu}$ as in the case of mean-centered perturbation method. Due to the explicit analytical expression of $\mathbf{d}_{\lambda_{j}}$ in terms of the derivative of the mass and stiffness matrices, expensive numerical differentiation of $\lambda_{j}(\mathbf{x})$ at each step is not needed. Moreover, for most $p_{\mathbf{X}}(\mathbf{x})$, a closed-form expression of $\mathbf{d}_{L}(\mathbf{x})$ is available. The form of Eq. (8) is very similar to that of Eq. (5). As mentioned before, when the basic random variables are non-Gaussian, determination of moments and the pdf is not straightforward. Some useful results for the case when $\mathbf{x}$ is Gaussian have been derived by Adhikari and Langley. ${ }^{19}$ In the next two sections a new method is proposed which is more suitable for non-Gaussian basic random variables.

\section{Method Based on the Asymptotic Integral}

\section{A. Multidimensional Integrals in Unbounded Domains}

In this section the moments of the eigenvalues are obtained based on an asymptotic approximation of the multidimensional integral. Consider a function $f(\mathbf{x}): \mathbb{R}^{m} \mapsto \mathbb{R}$ which is smooth and at least twice differentiable. Suppose we want to evaluate an integral of the following form:

$$
\mathcal{J}=\int_{\mathbb{R}^{m}} e^{-f(\mathbf{x})} d \mathbf{x}
$$

This is a $m$-dimensional integral over the unbounded domain $\mathbb{R}^{m}$. The maximum contribution to this integral comes from the neighborhood where $f(\mathbf{x})$ reaches its global minimum. Suppose that $f(\mathbf{x})$ reaches its global minimum at an unique point $\boldsymbol{\theta} \in \mathbb{R}^{m}$. Therefore, at $\mathbf{x}=\boldsymbol{\theta}$

$$
\frac{\partial f(\mathbf{x})}{\partial x_{k}}=0, \forall k \quad \text { or } \quad \mathbf{d}_{f}(\boldsymbol{\theta})=\mathbf{0} .
$$

Using this, expand $f(\mathbf{x})$ in a Taylor series about $\boldsymbol{\theta}$ and rewrite Eq. (13) as

$$
\begin{aligned}
\mathcal{J} & =\int_{\mathbb{R}^{m}} e^{-\left\{f(\boldsymbol{\theta})+\frac{1}{2}(\mathbf{x}-\boldsymbol{\theta})^{T} \mathbf{D}_{f}(\boldsymbol{\theta})(\mathbf{x}-\boldsymbol{\theta})+\varepsilon(\mathbf{x}, \boldsymbol{\theta})\right\}} d \mathbf{x} \\
& =e^{-f(\boldsymbol{\theta})} \int_{\mathbb{R}^{m}} e^{-\frac{1}{2}(\mathbf{x}-\boldsymbol{\theta})^{T} \mathbf{D}_{f}(\boldsymbol{\theta})(\mathbf{x}-\boldsymbol{\theta})-\varepsilon(\mathbf{x}, \boldsymbol{\theta})} d \mathbf{x}
\end{aligned}
$$


where $\varepsilon(\mathbf{x}, \boldsymbol{\theta})$ is the error if only the terms up to second-order were retained in the Taylor series expansion. With suitable scaling of $\mathbf{x}$ the integral in (13) can be transformed to the so called 'Laplace integral'. Under special conditions such integrals can be well approximated using asymptotic methods. The relevant mathematical methods and formal derivations are covered in detail in the books by Bleistein and Handelsman ${ }^{20}$ and Wong. ${ }^{21}$ Here we propose a somewhat different version of asymptotic integrals. The error $\varepsilon(\mathbf{x}, \boldsymbol{\theta})$ depends on higher of derivatives of $f(\mathbf{x})$ at $\mathbf{x}=\boldsymbol{\theta}$. If they are small compared to $f(\boldsymbol{\theta})$ their contribution will negligible to the value of the integral. So we assume that $f \boldsymbol{\theta})$ is large so that

$$
\left|\frac{1}{f(\boldsymbol{\theta})} \mathcal{D}^{(j)}(f(\boldsymbol{\theta}))\right| \rightarrow 0 \quad \text { for } \quad j>2
$$

where $\mathcal{D}^{(j)}(f(\boldsymbol{\theta}))$ is $j$ th order derivative of $f(\mathbf{x})$ evaluated at $\mathbf{x}=\boldsymbol{\theta}$. Under such assumptions it is easy to see that $\varepsilon(\mathbf{x}, \boldsymbol{\theta}) \rightarrow 0$. Therefore the integral in (15) can be approximated as

$$
\mathcal{J} \approx e^{-f(\boldsymbol{\theta})} \int_{\mathbb{R}^{m}} e^{-\frac{1}{2}(\mathbf{x}-\boldsymbol{\theta})^{T} \mathbf{D}_{f}(\boldsymbol{\theta})(\mathbf{x}-\boldsymbol{\theta})} d \mathbf{x}
$$

If $\boldsymbol{\theta}$ is the global minima of $f(\mathbf{x})$ in $\mathbb{R}^{m}$, the symmetric Hessian matrix $\mathbf{D}_{f}(\boldsymbol{\theta}) \in \mathbb{R}^{m \times m}$ is also expected to be positive definite. Now use the coordinate transformation

$$
\boldsymbol{\xi}=(\mathbf{x}-\boldsymbol{\theta}) \mathbf{D}_{f}^{-1 / 2}(\boldsymbol{\theta})
$$

The Jacobian of this transformation is

$$
\|\mathbf{J}\|=\left\|\mathbf{D}_{f}(\boldsymbol{\theta})\right\|^{-1 / 2}
$$

Using Eq. (18), the integral in Eq. (17) can be evaluated as

$$
\begin{aligned}
\mathcal{J} & \approx e^{-f(\boldsymbol{\theta})} \int_{\mathbb{R}^{m}}\left\|\mathbf{D}_{f}(\boldsymbol{\theta})\right\|^{-1 / 2} e^{-\frac{1}{2}\left(\boldsymbol{\xi}^{T} \boldsymbol{\xi}\right)} d \boldsymbol{\xi} \\
\text { or } \quad \mathcal{J} & \approx(2 \pi)^{m / 2} e^{-f(\boldsymbol{\theta})}\left\|\mathbf{D}_{f}(\boldsymbol{\theta})\right\|^{-1 / 2}
\end{aligned}
$$

A similar approach was outlined by Papadimitriou et. al. ${ }^{22}$ for the calculation of response moments of a dynamic system. This asymptotic analysis is somewhat different from the widely used Laplace's method of asymptotic approximation of integrals (Wong, ${ }^{21}$ Chapter IX, Theorem 3). Here it is assumed that $f(\boldsymbol{\theta})$ itself is a large quantity, unlike a free scaling parameter $(\lambda)$, as in the case of Laplace type integrals. Equation (21) will now be used to obtain moments of the eigenvalues.

\section{B. Calculation of an Arbitrary Moment of the Eigenvalues}

An arbitrary $r$ th order moment of the eigenvalues can be obtained from

$$
\begin{aligned}
\mu_{j}^{(r)}=\mathrm{E}\left[\lambda_{j}^{r}(\mathbf{x})\right] & =\int_{\mathbb{R}^{m}} \lambda_{j}^{r}(\mathbf{x}) p_{\mathbf{x}}(\mathbf{x}) d \mathbf{x} \\
& =\int_{\mathbb{R}^{m}} e^{-\left(L(\mathbf{x})-r \ln \lambda_{j}(\mathbf{x})\right)} d \mathbf{x}, \quad r=1,2,3 \cdots
\end{aligned}
$$

The equation can be expressed in the form of Eq. (13) by choosing

$$
f(\mathbf{x})=L(\mathbf{x})-r \ln \lambda_{j}(\mathbf{x})
$$

Differentiating the above equation with respect to $x_{k}$ we obtain

$$
\frac{\partial f(\mathbf{x})}{\partial x_{k}}=\frac{\partial L(\mathbf{x})}{\partial x_{k}}-\frac{r}{\lambda_{j}(\mathbf{x})} \frac{\partial \lambda_{j}(\mathbf{x})}{\partial x_{k}}
$$

The optimal point $\boldsymbol{\theta}$ can be obtained from (14) by equating the above expression to zero. Therefore at $\mathbf{x}=\boldsymbol{\theta}$

$$
\begin{gathered}
\frac{\partial f(\mathbf{x})}{\partial x_{k}}=0, \quad \forall k \\
\text { or } \frac{r}{\lambda_{j}(\boldsymbol{\theta})} \frac{\partial \lambda_{j}(\boldsymbol{\theta})}{\partial x_{k}}=\frac{\partial L(\boldsymbol{\theta})}{\partial x_{k}}, \quad \forall k \\
\text { or } \quad \mathbf{d}_{\lambda_{j}}(\boldsymbol{\theta}) r=\lambda_{j}(\boldsymbol{\theta}) \mathbf{d}_{L}(\boldsymbol{\theta})
\end{gathered}
$$


Equation (27) is very similar to Eq. (12) and needs to solved numerically to obtain $\boldsymbol{\theta}$. The elements of the Hessian matrix $\mathbf{D}_{f}(\boldsymbol{\theta})$ can be obtained by differentiating Eq. (24) with respect to $x_{l}$ :

$$
\begin{aligned}
\frac{\partial^{2} f(\mathbf{x})}{\partial x_{k} \partial x_{l}} & =\frac{\partial^{2} L(\mathbf{x})}{\partial x_{k} \partial x_{l}}-r\left(-\frac{1}{\lambda_{j}^{2}(\mathbf{x})} \frac{\partial \lambda_{j}(\mathbf{x})}{\partial x_{l}} \frac{\partial \lambda_{j}(\mathbf{x})}{\partial x_{k}}+\frac{1}{\lambda_{j}(\mathbf{x})} \frac{\partial^{2} \lambda_{j}(\mathbf{x})}{\partial x_{k} \partial x_{l}}\right) \\
& =\frac{\partial^{2} L(\mathbf{x})}{\partial x_{k} \partial x_{l}}+\frac{1}{r}\left\{\frac{r}{\lambda_{j}(\mathbf{x})} \frac{\partial \lambda_{j}(\mathbf{x})}{\partial x_{k}}\right\}\left\{\frac{r}{\lambda_{j}(\mathbf{x})} \frac{\partial \lambda_{j}(\mathbf{x})}{\partial x_{l}}\right\}-\frac{r}{\lambda_{j}(\mathbf{x})} \frac{\partial^{2} \lambda_{j}(\mathbf{x})}{\partial x_{k} \partial x_{l}}
\end{aligned}
$$

At $\mathbf{x}=\boldsymbol{\theta}$ we can use Eq. (26) so that Eq. (29) reads

$$
\left.\frac{\partial^{2} f(\mathbf{x})}{\partial x_{k} \partial x_{l}}\right|_{\mathbf{x}=\boldsymbol{\theta}}=\frac{\partial^{2} L(\boldsymbol{\theta})}{\partial x_{k} \partial x_{l}}+\frac{1}{r} \frac{\partial L(\boldsymbol{\theta})}{\partial x_{k}} \frac{\partial L(\boldsymbol{\theta})}{\partial x_{l}}-\frac{r}{\lambda_{j}(\boldsymbol{\theta})} \frac{\partial^{2} \lambda_{j}(\boldsymbol{\theta})}{\partial x_{k} \partial x_{l}} .
$$

Combining this equation for all $k$ and $l$ we have

$$
\mathbf{D}_{f}(\boldsymbol{\theta})=\mathbf{D}_{L}(\boldsymbol{\theta})+\frac{1}{r} \mathbf{d}_{L}(\boldsymbol{\theta}) \mathbf{d}_{L}(\boldsymbol{\theta})^{T}-\frac{r}{\lambda_{j}(\boldsymbol{\theta})} \mathbf{D}_{\lambda_{j}}(\boldsymbol{\theta})
$$

where $\mathbf{D}_{\lambda_{j}}(\bullet)$ is defined in Eq. (7). Using the asymptotic approximation (21), the $r$ th moment of the eigenvalues can be obtained as

$$
\mu_{j}^{(r)} \approx(2 \pi)^{m / 2} \lambda_{j}^{r}(\boldsymbol{\theta}) e^{-L(\boldsymbol{\theta})}\left\|\mathbf{D}_{L}(\boldsymbol{\theta})+\frac{1}{r} \mathbf{d}_{L}(\boldsymbol{\theta}) \mathbf{d}_{L}(\boldsymbol{\theta})^{T}-\frac{r}{\lambda_{j}(\boldsymbol{\theta})} \mathbf{D}_{\lambda_{j}}(\boldsymbol{\theta})\right\|^{-1 / 2}
$$

This is perhaps the most general formula to obtain the moments of the eigenvalues of linear stochastic dynamic systems. The optimal point $\boldsymbol{\theta}$ needs to be calculated by solving non-linear set of equations Eq. (27) for each $\lambda_{j}$ and $r$. If $\boldsymbol{\theta}$ is not unique then it is required to sum the contributions arising from all such optimal points separately. Several special cases arising from Eq. (32) are of practical interest:

- Random vector $\mathbf{x}$ has multivariate Gaussian distribution: In this case $L(\mathbf{x})$ is given by Eq. (3) and by differentiating this we obtain

$$
\begin{aligned}
\mathbf{d}_{L}(\mathbf{x}) & =\boldsymbol{\Sigma}^{-1} \mathbf{x} \\
\text { and } \quad \mathbf{D}_{L}(\mathbf{x}) & =\boldsymbol{\Sigma}^{-1}
\end{aligned}
$$

The optimal point $\boldsymbol{\theta}$ can be obtained from Eq. (27) as

$$
\boldsymbol{\theta}=\frac{r}{\lambda_{j}(\boldsymbol{\theta})} \boldsymbol{\Sigma} \mathbf{d}_{\lambda_{j}}(\boldsymbol{\theta})
$$

Using Eq. (33) and Eq. (34), the Hessian matrix can be derived from Eq. (31) as

$$
\begin{aligned}
\mathbf{D}_{f}(\boldsymbol{\theta}) & =\boldsymbol{\Sigma}^{-1}+\frac{1}{r} \boldsymbol{\Sigma}^{-1} \boldsymbol{\theta} \boldsymbol{\theta}^{T} \boldsymbol{\Sigma}^{-1}-\frac{r}{\lambda_{j}(\boldsymbol{\theta})} \mathbf{D}_{\lambda_{j}}(\boldsymbol{\theta}) \\
& =\boldsymbol{\Sigma}^{-1}\left(\mathbf{I}+\frac{1}{r} \boldsymbol{\theta} \boldsymbol{\theta}^{T} \boldsymbol{\Sigma}^{-1}\right)-\frac{r}{\lambda_{j}(\boldsymbol{\theta})} \mathbf{D}_{\lambda_{j}}(\boldsymbol{\theta})
\end{aligned}
$$

Therefore, the $r$ th moment of the eigenvalues can be obtained from Eq. (32) as

$$
\mu_{j}^{(r)} \approx \lambda_{j}^{r}(\boldsymbol{\theta}) e^{-\frac{1}{2}(\boldsymbol{\theta}-\boldsymbol{\mu})^{T} \boldsymbol{\Sigma}^{-1}(\boldsymbol{\theta}-\boldsymbol{\mu})}\|\boldsymbol{\Sigma}\|^{-1 / 2}\left\|\mathbf{D}_{f}(\boldsymbol{\theta})\right\|^{-1 / 2}
$$

Using Eq. (36) and recalling that for any two matrices $\mathbf{A}$ and $\mathbf{B},\|\mathbf{A}\|\|\mathbf{B}\|=\|\mathbf{A B}\|$ we have

$$
\mu_{j}^{(r)} \approx \lambda_{j}^{r}(\boldsymbol{\theta}) e^{-\frac{1}{2}(\boldsymbol{\theta}-\boldsymbol{\mu})^{T} \boldsymbol{\Sigma}^{-1}(\boldsymbol{\theta}-\boldsymbol{\mu})}\left\|\mathbf{I}+\frac{1}{r} \boldsymbol{\theta} \boldsymbol{\theta}^{T} \boldsymbol{\Sigma}^{-1}-\frac{r}{\lambda_{j}(\boldsymbol{\theta})} \boldsymbol{\Sigma D}_{\lambda_{j}}(\boldsymbol{\theta})\right\|^{-1 / 2} .
$$

For the standardized Gaussian random vector $\boldsymbol{\mu}=\mathbf{0}$ and $\boldsymbol{\Sigma}=\mathbf{I}$. In this case Eq. (38) reduces to the corresponding equation derived previously by Adhikari and Langley. ${ }^{19}$ 
- Mean of the eigenvalues: The mean of the eigenvalues can be obtained by substituting $r=1$ in Eq. (32), that is

$$
\widehat{\lambda}_{j}=\mu_{j}^{(1)}=\lambda_{j}(\boldsymbol{\theta}) e^{-L(\boldsymbol{\theta})}\left\|\mathbf{D}_{L}(\boldsymbol{\theta})+\mathbf{d}_{L}(\boldsymbol{\theta}) \mathbf{d}_{L}(\boldsymbol{\theta})^{T}-\mathbf{D}_{\lambda_{j}}(\boldsymbol{\theta}) / \lambda_{j}(\boldsymbol{\theta})\right\|^{-1 / 2} .
$$

- Central moments of the eigenvalues: Once the mean in known, the central moments can be expressed in terms of the raw moments $\mu_{j}^{(r)}$ using the binomial transform

$$
\mathrm{E}\left[\left(\lambda_{j}-\widehat{\lambda}_{j}\right)^{r}\right]=\sum_{k=0}^{r}\left(\begin{array}{l}
r \\
k
\end{array}\right)(-1)^{r-k} \mu_{j}^{(k)} \widehat{\lambda}_{j}^{r-k} .
$$

The probability density function of the eigenvalues is considered in the next section.

\section{Probability Density Function of the Eigenvalues}

\section{A. Maximum Entropy Probability Density Function}

Once the moments of the eigenvalues are known, the pdf of the eigenvalues can be obtained using maximum entropy method (MEM). Since Eq. (32) can be used to calculate any arbitrary order moment, the pdf can be obtained very accurately by taking higher order moments. Here, following Kapur and Kesavan, ${ }^{23}$ a general approach is presented.

Since it is assumed that $\mathbf{M}$ and $\mathbf{K}$ are symmetric and positive definite random matrices, all the eigenvalues are real and positive. Suppose the pdf of $\lambda_{j}$ is given by $p_{\lambda_{j}}(u)$ where $u$ is positive, that is $u \in[0, \infty]$. Considering that only first $n$ moments are used, the pdf of each eigenvalue must satisfy the following constraints:

$$
\int_{0}^{\infty} p_{\lambda_{j}}(u) d u=1
$$

and

$$
\int_{0}^{\infty} u^{r} p_{\lambda_{j}}(u) d u=\mu_{j}^{(r)}, \quad r=1,2,3, \cdots, n .
$$

Using Shannon's measure of entropy

$$
\mathcal{S}=-\int_{0}^{\infty} p_{\lambda_{j}}(u) \ln p_{\lambda_{j}}(u) d u
$$

we construct the Lagrangian

$$
\mathcal{L}=-\int_{0}^{\infty} p_{\lambda_{j}}(u) \ln p_{\lambda_{j}}(u) d u-\left(\rho_{0}-1\right)\left[\int_{0}^{\infty} p_{\lambda_{j}}(u) d u-1\right]-\sum_{r=1}^{n} \rho_{r}\left[\int_{0}^{\infty} u^{r} p_{\lambda_{j}}(u) d u-\mu_{j}^{(r)}\right]
$$

where $\rho_{r}, r=0,1,2, \cdots, n$ are Lagrange multipliers. The function $p_{\lambda_{j}}(u)$ which maximizes $\mathcal{L}$ can be obtained using the calculus of variations. Using the Euler-Lagrange equation the solution is given by

$$
p_{\lambda_{j}}(u)=e^{-\left\{\rho_{0}+\sum_{i=1}^{n} \rho_{i} u^{i}\right\}}=e^{-\rho_{0}} e^{-\sum_{i=1}^{n} \rho_{i} u^{i}}, \quad u \geq 0 .
$$

The Lagrange multipliers can be obtained from the constraint equations (41) and (42) as

$$
e^{\rho_{0}}=\int_{0}^{\infty} e^{-\sum_{i=1}^{n} \rho_{i} u^{i}} d u
$$

and

$$
e^{\rho_{0}} \mu_{j}^{(r)}=\int_{0}^{\infty} u^{r} e^{-\sum_{i=1}^{n} \rho_{i} u^{i}} d u, \quad \text { for } \quad r=0,1,2, \cdots n .
$$

Closed-form expressions for $\rho_{r}$ are in general not possible for all $n$. If we take $n=2$, then the resulting pdf can be expressed by a truncated Gaussian density function

$$
p_{\lambda_{j}}(u)=\frac{1}{\sqrt{2 \pi} \sigma_{j} \Phi\left(\hat{\lambda}_{j} / \sigma_{j}\right)} e^{-\frac{\left(u-\hat{\lambda}_{j}\right)^{2}}{2 \sigma_{j}^{2}}}, u \geq 0
$$

where $\sigma_{j}$ is given by

$$
\sigma_{j}^{2}=\mu_{j}^{(2)}-\widehat{\lambda}_{j}^{2}
$$

This truncated Gaussian density function ensures that the probability of any eigenvalues becoming negative is zero. 


\section{B. Approximation by $\chi^{2}$ Probability Density Function}

We use an approximation analogous to Pearson's ${ }^{24}$ three moment central $\chi^{2}$ approximation to the distribution of a noncentral $\chi^{2}$. The eigenvalues are approximated as

$$
p_{\lambda_{j}}(u) \approx \eta_{j}+\gamma_{j} \chi_{\nu_{j}}^{2}(u) .
$$

The constants $\eta_{j}, \gamma_{j}$, and $\nu_{j}$ are obtained such that the first three moments of $\lambda_{j}$ are equal to that of the approximated $\chi^{2}$ pdf. The moment generating function of the approximated $\chi^{2}$ pdf is given by

$$
\mathrm{E}\left[e^{-s\left(\eta_{j}+\gamma_{j} \chi_{\nu_{j}}^{2}\right)}\right]=e^{-s \eta_{j}}\left(1+2 s \gamma_{j}\right)^{-\nu_{j} / 2} .
$$

Equating the first three moments we have

$$
\begin{aligned}
& \eta_{j}+\nu_{j} \gamma_{j}=\mu_{j}^{(1)}, \\
& \eta_{j}{ }^{2}+2 \eta_{j} \nu_{j} \gamma_{j}+\nu_{j}{ }^{2} \gamma_{j}{ }^{2}+2 \nu_{j} \gamma_{j}{ }^{2}=\mu_{j}^{(2)} \\
& \eta_{j}{ }^{3}+3 \eta_{j}{ }^{2} \nu_{j} \gamma_{j}+3 \eta_{j} \nu_{j}{ }^{2} \gamma_{j}{ }^{2}+6 \eta_{j} \nu_{j} \gamma_{j}{ }^{2}+\nu_{j}{ }^{3} \gamma_{j}{ }^{3}+6 \nu_{j}{ }^{2} \gamma_{j}{ }^{3}+8 \nu_{j} \gamma_{j}{ }^{3}=\mu_{j}^{(3)} .
\end{aligned}
$$

This set of coupled non-linear equations can be solved exactly in closed-form to obtain $\eta_{j}, \gamma_{j}$, and $\nu_{j}$ :

$$
\begin{aligned}
\eta_{j} & =\frac{\mu_{j}^{(1)^{2}} \mu_{j}^{(2)}-2 \mu_{j}^{(2)^{2}}+\mu_{j}^{(1)} \mu_{j}^{(3)}}{2 \mu_{j}^{(1)^{3}}-3 \mu_{j}^{(1)} \mu_{j}^{(2)}+\mu_{j}^{(3)}} \\
\gamma_{j} & =\frac{2 \mu_{j}^{(1)^{3}}-3 \mu_{j}^{(1)} \mu_{j}^{(2)}+\mu_{j}^{(3)}}{4\left(\mu_{j}^{(2)}-\mu_{j}^{(1)^{2}}\right)}, \\
\text { and } \nu_{j} & =8 \frac{\left(\mu_{j}^{(2)}-\mu_{j}^{(1)^{2}}\right)^{3}}{\left(2 \mu_{j}^{(1)^{3}}-3 \mu_{j}^{(1)} \mu_{j}^{(2)}+\mu_{3}^{\prime}\right)^{2}} .
\end{aligned}
$$

Moments of $\lambda_{j}(\mathbf{x})$ obtained in Eq. (32), can be used directly in the right-hand side of these equations. Using the transformation in (50) the approximate probability density function of $\lambda_{j}(\mathbf{x})$ is given by

$$
p_{\lambda_{j}}(u) \approx \frac{1}{\gamma_{j}} p_{\chi_{\nu_{j}}}\left(\frac{u-\eta_{j}}{\gamma_{j}}\right)=\frac{\left(u-\eta_{j}\right)^{\nu_{j} / 2-1} e^{-\left(u-\eta_{j}\right) / 2 \gamma_{j}}}{\left(2 \gamma_{j}\right)^{\nu_{j} / 2} \Gamma\left(\nu_{j} / 2\right)} .
$$

It should be noted that given the moments, several pdfs can be fitted. The two approximated pdf proposed here have simple forms. Application of the approximate pdfs derived here is illustrated in the next section.

\section{Application Example}

\section{A. Model System and Computational Methodology}

A three-degree-of-freedom undamped spring-mass system, taken from reference, ${ }^{25}$ is shown in Fig. 1. The eigenvalue problem associated with this system is considered to illustrate a possible application of the expressions developed in the paper. The mass and stiffness matrices of the example system are given by

$$
\mathbf{M}=\left[\begin{array}{ccc}
m_{1} & 0 & 0 \\
0 & m_{2} & 0 \\
0 & 0 & m_{3}
\end{array}\right] \quad \text { and } \quad \mathbf{K}=\left[\begin{array}{ccc}
k_{1}+k_{4}+k_{6} & -k_{4} & -k_{6} \\
-k_{4} & k_{4}+k_{5}+k_{6} & -k_{5} \\
-k_{6} & -k_{5} & k_{5}+k_{3}+k_{6}
\end{array}\right] \text {. }
$$

It is assumed that all mass and stiffness constants are random. The randomness in these parameters are assumed to be of the following form:

$$
\begin{array}{ll}
m_{i}=\bar{m}_{i}\left(1+\epsilon_{m} x_{i}\right), & i=1,2,3 \\
k_{i}=\bar{k}_{i}\left(1+\epsilon_{k} x_{i+3}\right), & i=1, \cdots, 6 .
\end{array}
$$

Here $\mathbf{x}=\left\{x_{1}, \cdots, x_{9}\right\}^{T} \in \mathbb{R}^{9}$ is the vector of random variables. It is assumed that all random variables are Gaussian and uncorrelated with zero mean and unit standard deviation. Therefore, the mean values of $m_{i}$ and $k_{i}$ are given by $\bar{m}_{i}$ 


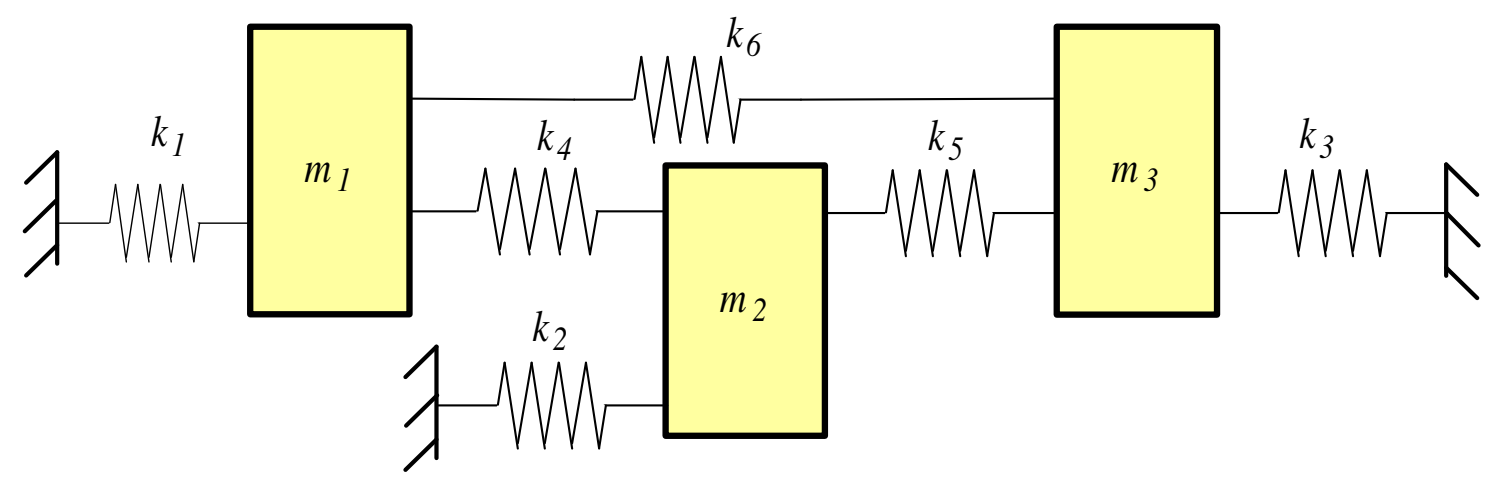

Figure 1 The three degree-of-freedom random system

and $\bar{k}_{i}$. The numerical values of both the 'strength parameters' $\epsilon_{m}$ and $\epsilon_{k}$ are fixed at 0.15 . The strength parameters are selected so that the system matrices are almost surely positive definite. In order to obtain statistics of the eigenvalues using the methods developed in this paper the gradient vector and the Hessian matrix of the eigenvalues are required. As shown in Appendix A, this in turn requires the derivative of the system matrices with respect to the entries of $\mathbf{x}$. For most practical problems, which usually involve Finite Element modeling, these derivatives need to be determined numerically. However, for this simple example the derivatives can be obtained in closed-form and they are given in Appendix B.

We calculate the moments and the probability density functions of the three eigenvalues of the system. Recall that the eigenvalues obtained from Eq. (1) are the square of the natural frequencies $\left(\lambda_{j}=\omega_{j}^{2}\right)$. The following two sets of physically meaningful parameter values are considered:

- Case 1: All eigenvalues are well separated

For this case $\bar{m}_{i}=1.0 \mathrm{~kg}$ for $i=1,2,3 ; \bar{k}_{i}=1.0 \mathrm{~N} / \mathrm{m}$ for $i=1, \cdots, 5$ and $k_{6}=3.0 \mathrm{~N} / \mathrm{m}$.

- Case 2: Two eigenvalues are very close

All parameter values are the same except $k_{6}=1.275 \mathrm{~N} / \mathrm{m}$.

The moments of the eigenvalues for the above two cases are calculated from Eq. (38) with $\boldsymbol{\mu}=\mathbf{0}$ and $\boldsymbol{\Sigma}=\mathbf{I}$. A commercial non-linear equation solver is used to obtain the optimal point $\boldsymbol{\theta}$ from Eq. (35). The moments are then used to obtain $\sigma_{j}$ from Eq. (49) and the constants in Eqs. (55)-(57). Using these constants the truncated Gaussian pdf and the $\chi^{2}$ pdf of the eigenvalues are obtained from Eqs. (48) and (58) respectively. These results are compared with Monte Carlo simulation. The samples of the nine independent Gaussian random variables $x_{i}, i=1, \cdots, 9$ are generated and the eigenvalues are computed directly from Eq. (1). A total of 5000 samples are used to obtain the statistical moments and histograms of the pdf of the eigenvalues. The results obtained from Monte Carlo simulation are assumed to be the benchmark for the purpose of comparing the analytical methods proposed in this paper. For the purpose of determining the accuracy of the proposed methods, we calculate the percentage error associated with an arbitrary $r$ th moment given by

$$
\text { Error }=\frac{\mu^{(r)}-\left\{\mu^{(r)}\right\}_{\mathrm{MCS}}}{\left\{\mu^{(r)}\right\}_{\mathrm{MCS}}} \times 100 .
$$

The results for the two cases are presented and discussed in the next subsection.

\section{B. Numerical Results}

\section{B.1. Case 1: All eigenvalues are well separated}

When all of the eigenvalues are well separated their derivatives with respect to the system parameters generally behave well. For the given parameter values the eigenvalues of the corresponding deterministic system is given by

$$
\bar{\lambda}_{1}=1, \quad \bar{\lambda}_{2}=4, \quad \text { and } \quad \bar{\lambda}_{3}=8
$$

The random 'scatter' of the eigenvalues is shown in Fig. 2 for 1000 samples from the Monte Carlo simulation. It can be seen that the highest eigenvalue has the maximum scatter and because the eigenvalues are well separated, there is very little statistical overlap between them. Figure 3 shows the percentage error for the first four moments of the eigenvalues. These errors are calculated from Eq. (62) and they are reasonably small considering that the strength of randomness for all nine random variables are $15 \%$. Note that error associated with higher eigenvalues are higher and also for a fixed eigenvalue, the higher order moments have more errors. 


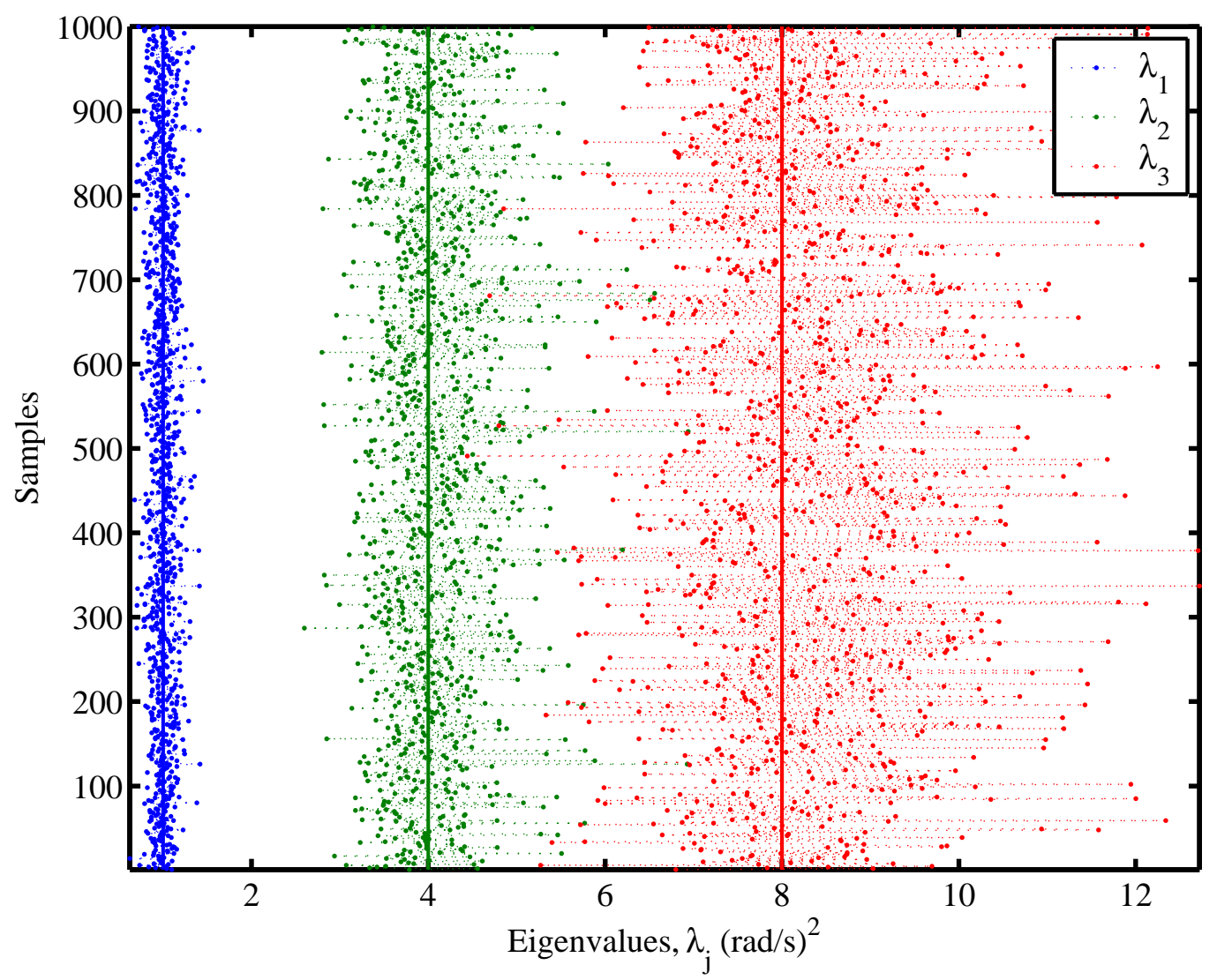

Figure 2 Statistical scatter in the eigenvalues; Case 1.

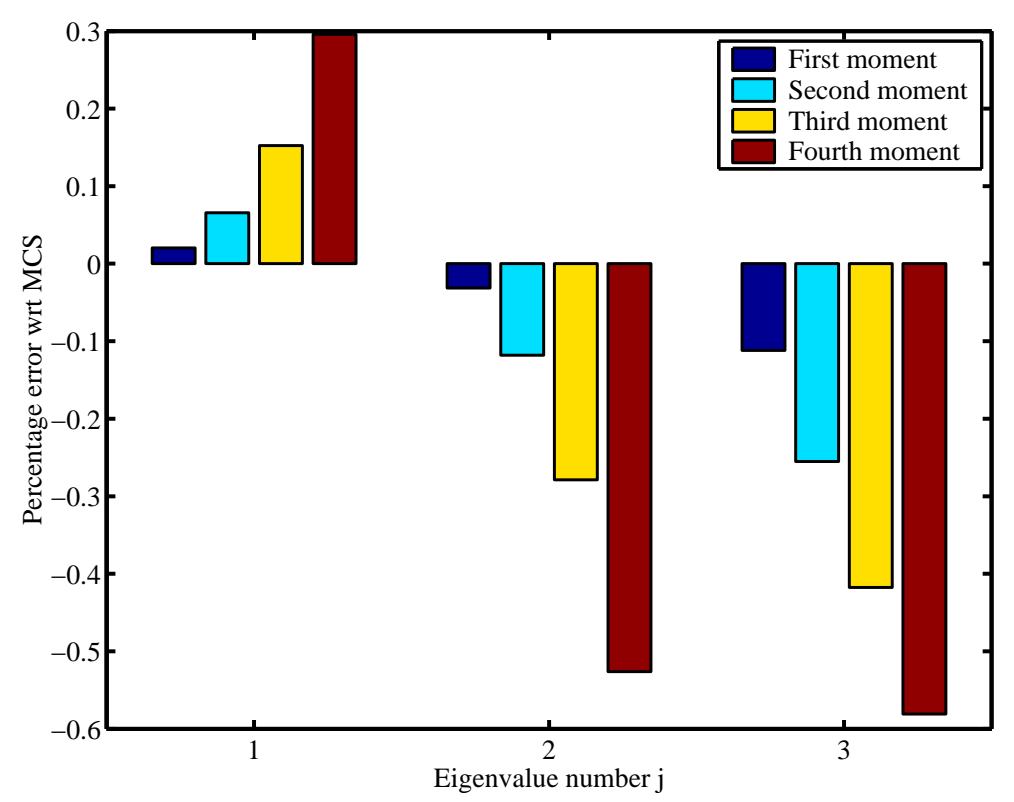

Figure 3 Percentage error for first four moments of the eigenvalues; Case 1.

Now consider the probability density function of the eigenvalues. The pdf of the first eigenvalue obtained from the two methods are shown in Fig. 4. On the same plot, normalized histograms of the eigenvalue obtained from the Monte Carlo simulation are also shown. Both approximate methods match well with the Monte Carlo simulation result. This is expected since the first three moments are obtained very accurately (less than $0.2 \%$ error as seen in Fig. 3). The probability density functions of the second and third eigenvalues are shown in Fig. 5. The region of statistical overlap is indeed small and can be verified from the plot of the actual samples in Fig. 2. Again, both approximate methods 


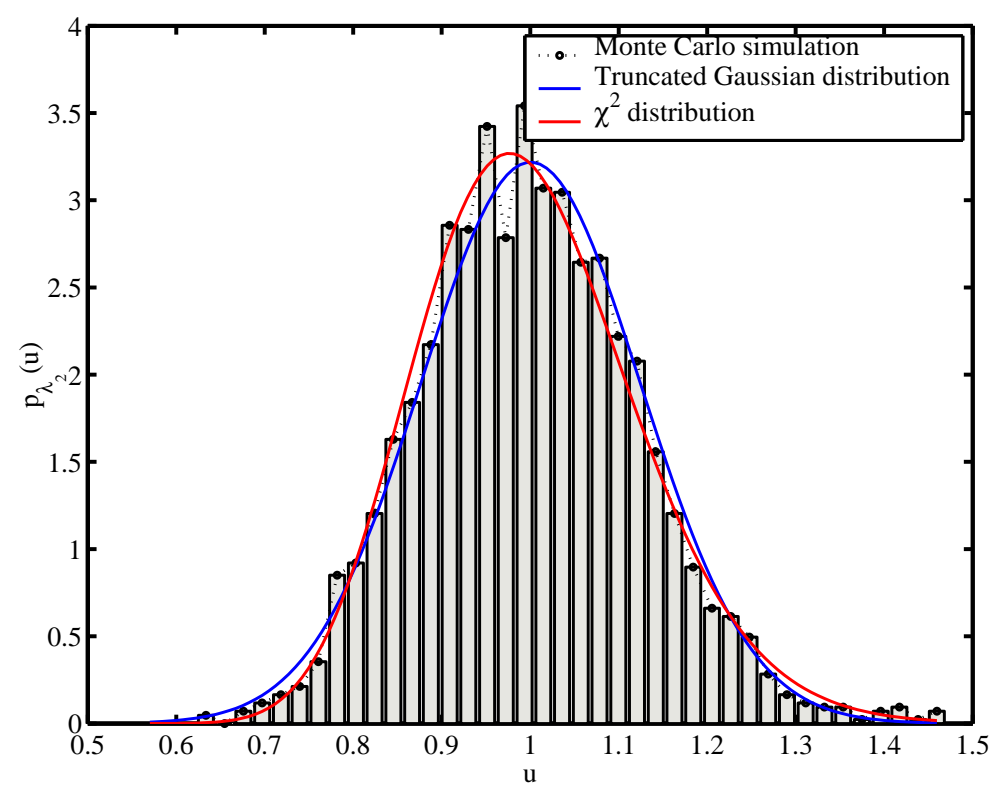

Figure 4 Probability density function of the first eigenvalue; Case 1.

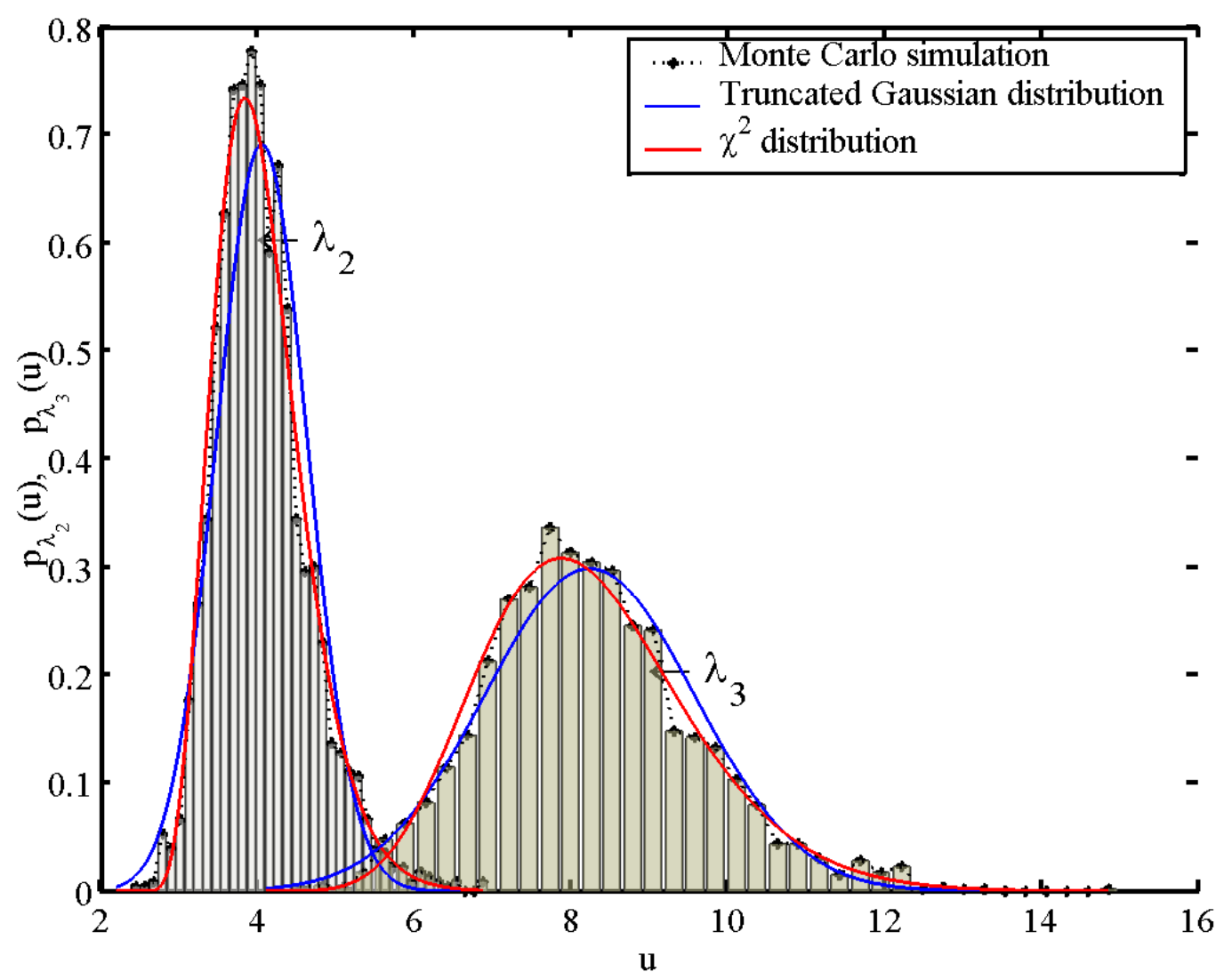

Figure 5 Probability density functions of the second and third eigenvalues; Case 1.

match well with the Monte Carlo simulation result.

\section{B.2. Case 2: Two eigenvalues are very close}

When some eigenvalues are closely spaced, their derivatives with respect to the system parameters may badly behave. ${ }^{25}$ Indeed, if repeated eigenvalues exist, the formulation proposed here breaks down. The purpose of studying this case is to investigate how the proposed methods work when there are closely spaced eigenvalues so that there is 
a significant statistical overlap between them. For the given parameter values the eigenvalues of the corresponding deterministic system are calculated as

$$
\bar{\lambda}_{1}=1, \quad \bar{\lambda}_{2}=4, \quad \text { and } \quad \bar{\lambda}_{3}=4.55
$$

Clearly $\bar{\lambda}_{2}$ and $\bar{\lambda}_{3}$ are very close to each other. The random 'scatter' of the eigenvalues is shown in Fig. 6 for 1000 samples from the Monte Carlo simulation. It can be seen that the third eigenvalue has the maximum scatter and

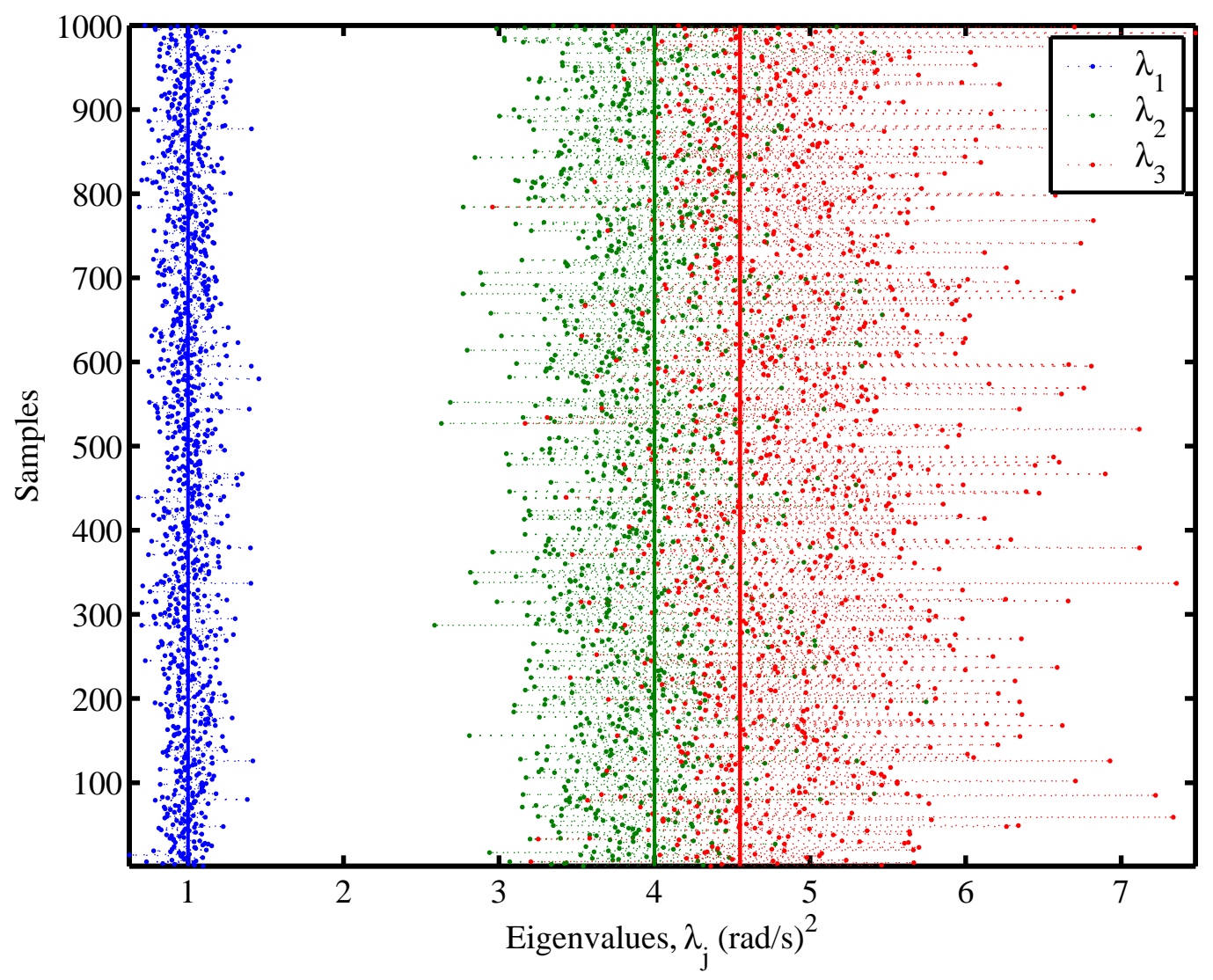

Figure 6 Statistical scatter in the eigenvalues; Case 2.

because the second and the third eigenvalues are close there is significant statistical overlap between them. Figure 7 shows the percentage error for the first four moments of the eigenvalues. These errors are calculated from Eq. (62). The general trend of these errors are similar to the previous case except that the magnitudes of the errors corresponding to second and third eigenvalues are higher. This is expected because these two eigenvalues are close to each other.

The probability density function of the first eigenvalue obtained from the two methods are shown in Fig. 8. On the same plot, normalized histograms of the eigenvalue obtained from Monte Carlo simulation are also shown. As in the previous case, both approximate methods match well with the Monte Carlo simulation result. This is expected since the first three moments are obtained very accurately for this case also. The probability density functions of the second and third eigenvalues are shown in Fig. 9. There is a significant region of statistical overlap which can also be verified from the plot of the actual samples in Fig. 6. In this case the truncated Gaussian density function performs better than the $\chi^{2}$ density function. However, none of the approximate methods match the Monte Carlo simulation result as well as in the previous case.

\section{Conclusions}

The statistics of the eigenvalues of linear dynamic systems with parameter uncertainties have been considered. It is assumed that the mass and stiffness matrices are smooth and at least twice differentiable functions of a set of random variables. The random variables are in general assumed to be non-Gaussian. The usual assumption of small randomness employed in most mean-centered based perturbation analysis is not employed in this study. A method based on asymptotic approximation of multidimensional integrals is proposed to obtain moments of the eigenvalues. The method is based on an unconstrained optimization problem. A closed-form expression of general $r$ th order moment 


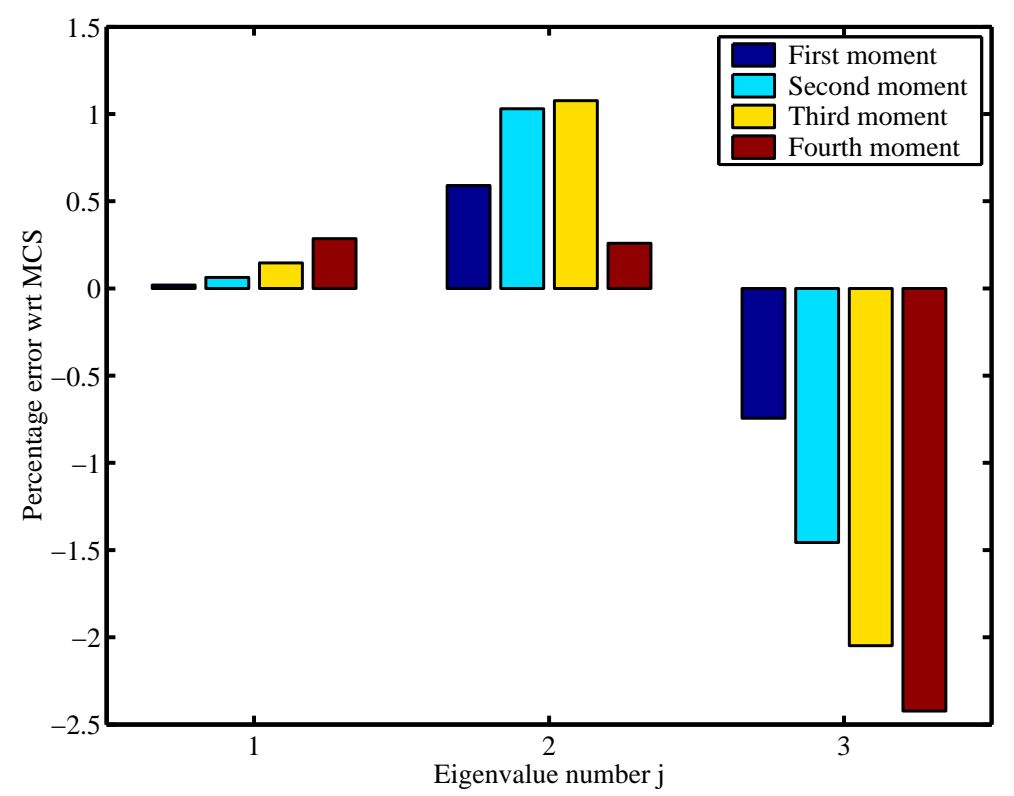

Figure 7 Percentage error for first four moments of the eigenvalues; Case 2.

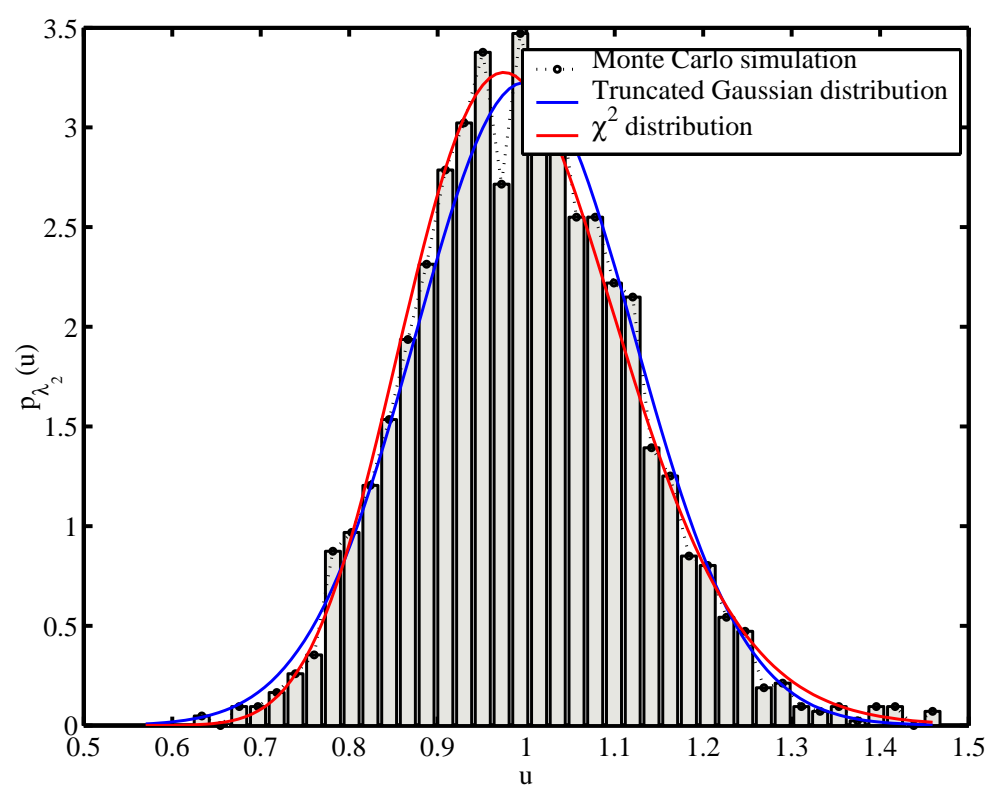

Figure 8 Probability density function of the first eigenvalue; Case 2.

of the eigenvalues was derived. Two simple approximations for the probability density function of the eigenvalues are derived. One is in terms of a truncated Gaussian random variable obtained using the maximum entropy principle. The other is a $\chi^{2}$ random variable approximation based on matching the first three moments of the eigenvalues. Both formulations yield closed-form expressions of the pdf which can be computed easily.

The proposed formulae are applied to a three-degree-of-freedom stochastic system. The moments and the probability density functions match well with the corresponding Monte Carlo simulation results when the eigenvalues are well separated. However, when some eigenvalues are closely spaced, the proposed methods do not produce very accurate results. Future studies will address the problem of closely spaced or repeated eigenvalues. Further research is also required to develop new methods to obtain joint probability density functions of the eigenvalues.

\section{Appendix A: Gradient Vector and Hessian Matrix of the Eigenvalues}

The eigenvectors of symmetric linear systems are orthogonal with respect to the mass and stiffness matrices. Normalize the eigenvectors so that they are unity mass normalized, that is,

$$
\phi_{j}^{T} \mathbf{M} \phi_{j}=1 .
$$




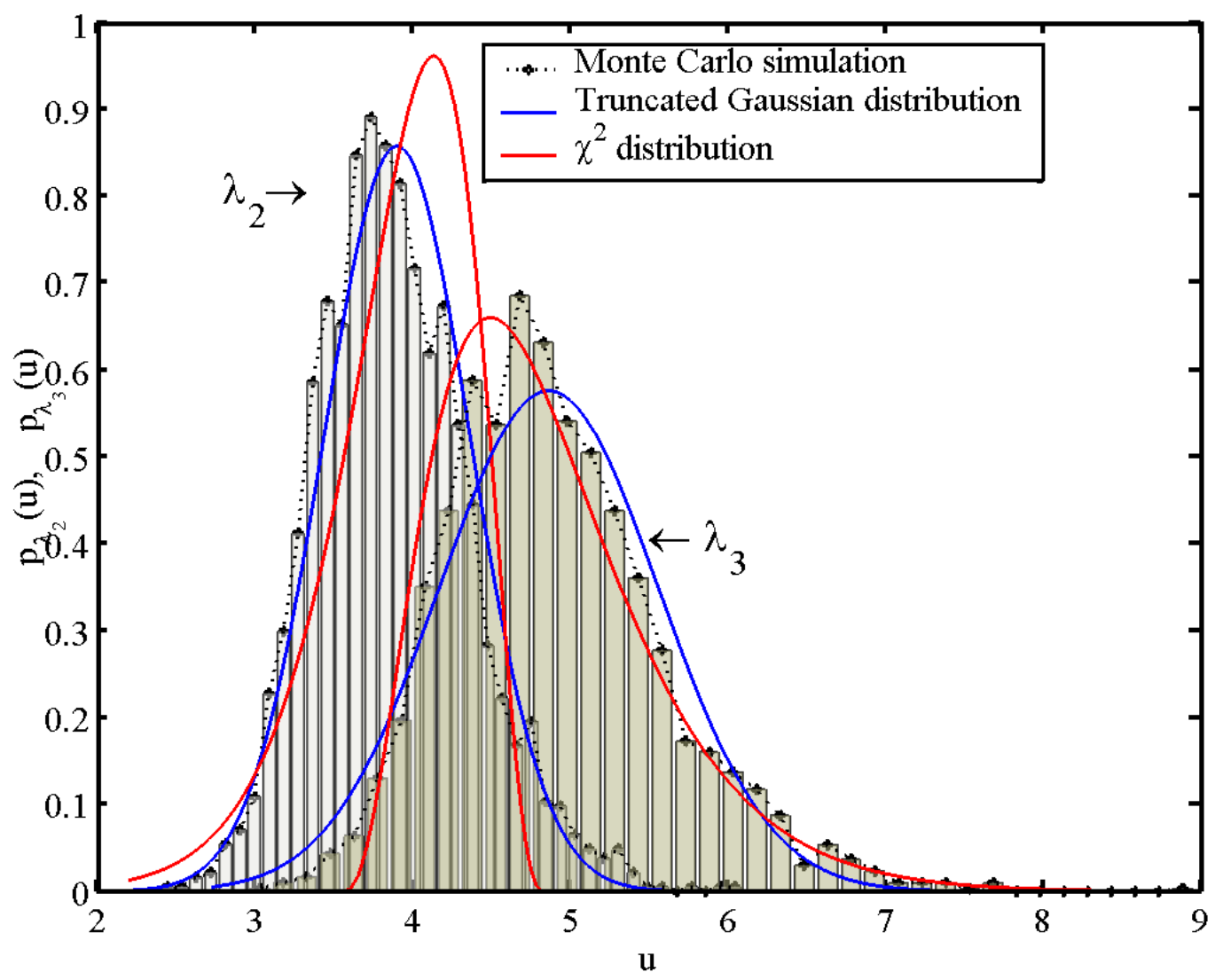

Figure 9 Probability density functions of the second and third eigenvalues; Case 2.

Using this and differentiating Eq. (1) with respect to $x_{k}$ it can be shown that ${ }^{26}$ for any $\mathbf{x}$

$$
\frac{\partial \lambda_{j}(\mathbf{x})}{\partial x_{k}}=\phi_{j}(\mathbf{x})^{T} \mathcal{G}_{j k}(\mathbf{x}) \phi_{j}(\mathbf{x})
$$

where

$$
\mathcal{G}_{j k}(\mathbf{x})=\left[\frac{\partial \mathbf{K}(\mathbf{x})}{\partial x_{k}}-\lambda_{j}(\mathbf{x}) \frac{\partial \mathbf{M}(\mathbf{x})}{\partial x_{k}}\right] .
$$

Differentiating Eq. (1) with respect to $x_{k}$ and $x_{l}$ Plaut and Huseyin ${ }^{27}$ have shown that, providing the eigenvalues are distinct,

$$
\begin{gathered}
\frac{\partial^{2} \lambda_{j}(\mathbf{x})}{\partial x_{k} \partial x_{l}}=\boldsymbol{\phi}_{j}(\mathbf{x})^{T}\left[\frac{\partial^{2} \mathbf{K}(\mathbf{x})}{\partial x_{k} \partial x_{l}}-\lambda_{j}(\mathbf{x}) \frac{\partial^{2} \mathbf{M}(\mathbf{x})}{\partial x_{k} \partial x_{l}}\right] \boldsymbol{\phi}_{j}(\mathbf{x})-\left(\boldsymbol{\phi}_{j}(\mathbf{x})^{T} \frac{\partial \mathbf{M}(\mathbf{x})}{\partial x_{k}} \boldsymbol{\phi}_{j}(\mathbf{x})\right)\left(\boldsymbol{\phi}_{j}(\mathbf{x})^{T} \mathcal{G}_{j l}(\mathbf{x}) \boldsymbol{\phi}_{j}(\mathbf{x})\right) \\
-\left(\boldsymbol{\phi}_{j}(\mathbf{x})^{T} \frac{\partial \mathbf{M}(\mathbf{x})}{\partial x_{l}} \boldsymbol{\phi}_{j}(\mathbf{x})\right)\left(\boldsymbol{\phi}_{j}(\mathbf{x})^{T} \mathcal{G}_{j k}(\mathbf{x}) \boldsymbol{\phi}_{j}(\mathbf{x})\right)+2 \sum_{r=1}^{N} \frac{\left(\boldsymbol{\phi}_{r}(\mathbf{x})^{T} \mathcal{G}_{j k}(\mathbf{x}) \boldsymbol{\phi}_{j}(\mathbf{x})\right)\left(\phi_{r}(\mathbf{x})^{T} \mathcal{G}_{j l}(\mathbf{x}) \boldsymbol{\phi}_{j}(\mathbf{x})\right)}{\lambda_{j}(\mathbf{x})-\lambda_{r}(\mathbf{x})} .
\end{gathered}
$$

Equations (66) and (68) completely define the elements of the gradient vector and Hessian matrix of the eigenvalues.

\section{Appendix B: Derivative of the System Matrices With Respect to the Random Variables}

The derivatives of $\mathbf{M}(\mathbf{x})$ and $\mathbf{K}(\mathbf{x})$ with respect to elements of $\mathbf{x}$ can be obtained from Eq. (59) together with Eqs. (60) and (61). For the mass matrix we have

$$
\frac{\partial \mathbf{M}}{\partial x_{1}}=\left[\begin{array}{ccc}
\bar{m}_{1} \epsilon_{m} & 0 & 0 \\
0 & 0 & 0 \\
0 & 0 & 0
\end{array}\right], \quad \frac{\partial \mathbf{M}}{\partial x_{2}}=\left[\begin{array}{ccc}
0 & 0 & 0 \\
0 & \bar{m}_{2} \epsilon_{m} & 0 \\
0 & 0 & 0
\end{array}\right], \quad \frac{\partial \mathbf{M}}{\partial x_{3}}=\left[\begin{array}{ccc}
0 & 0 & 0 \\
0 & 0 & 0 \\
0 & 0 & \bar{m}_{3} \epsilon_{m}
\end{array}\right] .
$$


All other $\frac{\partial \mathbf{M}}{\partial x_{i}}$ are null matrices. For the derivative of the stiffness matrix

$$
\begin{aligned}
& \frac{\partial \mathbf{K}}{\partial x_{4}}=\left[\begin{array}{ccc}
\bar{k}_{1} \epsilon_{k} & 0 & 0 \\
0 & 0 & 0 \\
0 & 0 & 0
\end{array}\right], \quad \frac{\partial \mathbf{K}}{\partial x_{5}}=\left[\begin{array}{ccc}
0 & 0 & 0 \\
0 & \bar{k}_{2} \epsilon_{k} & 0 \\
0 & 0 & 0
\end{array}\right], \quad \frac{\partial \mathbf{M}}{\partial x_{6}}=\left[\begin{array}{ccc}
0 & 0 & 0 \\
0 & 0 & 0 \\
0 & 0 & \bar{k}_{3} \epsilon_{k}
\end{array}\right] \\
& \frac{\partial \mathbf{K}}{\partial x_{7}}=\left[\begin{array}{ccc}
\bar{k}_{4} \epsilon_{k} & -\bar{k}_{4} \epsilon_{k} & 0 \\
-\bar{k}_{4} \epsilon_{k} & \bar{k}_{4} \epsilon_{k} & 0 \\
0 & 0 & 0
\end{array}\right], \quad \frac{\partial \mathbf{K}}{\partial x_{8}}=\left[\begin{array}{ccc}
0 & 0 & 0 \\
0 & \bar{k}_{5} \epsilon_{k} & -\bar{k}_{5} \epsilon_{k} \\
0 & -\bar{k}_{5} \epsilon_{k} & \bar{k}_{5} \epsilon_{k}
\end{array}\right], \quad \frac{\partial \mathbf{M}}{\partial x_{9}}=\left[\begin{array}{ccc}
\bar{k}_{6} \epsilon_{k} & 0 & -\bar{k}_{6} \epsilon_{k} \\
0 & 0 & 0 \\
-\bar{k}_{6} \epsilon_{k} & 0 & \bar{k}_{6} \epsilon_{k}
\end{array}\right]
\end{aligned}
$$

and all other $\frac{\partial \mathbf{K}}{\partial x_{i}}$ are null matrices. Also note that all of the first-order derivative matrices are independent of $\mathbf{x}$. For this reason, all the higher order derivatives of the $\mathbf{M}(\mathbf{x})$ and $\mathbf{K}(\mathbf{x})$ matrices are null matrices.

\section{References}

1 Boyce, W. E., Random Eigenvalue Problems, Probabilistic methods in applied mathematics, Academic Press, New York, 1968.

2 Scheidt, J. V. and Purkert, W., Random Eigenvalue Problems, North Holland, New York, 1983.

3 Ibrahim, R. A., "Structural dynamics with parameter uncertainties," Applied Mechanics Reviews, ASME, Vol. 40, No. 3, 1987, pp. $309-328$.

4 Benaroya, H. and Rehak, M., "Finite element methods in probabilistic structural analysis: a selective review," Applied Mechanics Reviews, ASME, Vol. 41, No. 5, 1988, pp. 201-213.

5 Benaroya, H., "Random eigenvalues, algebraic methods and structural dynamic models," Applied Mathematics and Computation, Vol. 52, 1992, pp. 37-66.

6 Manohar, C. S. and Ibrahim, R. A., "Progress in structural dynamics with stochastic parameter variations: 1987 to 1998," Applied Mechanics Reviews, ASME, Vol. 52, No. 5, May 1999, pp. 177-197.

7 Manohar, C. S. and Gupta, S., "Modeling and evaluation of structural reliability: Current status and future directions," Research reviews in structural engineering, Golden Jubilee Publications of Department of Civil Engineering, Indian Institute of Science, Bangalore, edited by K. S. Jagadish and R. N. Iyengar, Indian Institute of Science, Bangalore, University Press, Hyderabad, India, 2003.

8 Mehta, M. L., Random Matrices, Academic Press, San Diego, CA, 2nd ed., 1991.

9 Collins, J. D. and Thomson, W. T., "The eigenvalue problem for structural systems with statistical properties," AIAA Journal, Vol. 7, No. 4, April 1969, pp. 642-648.

10 Ramu, S. A. and Ganesan, R., "Stability analysis of a stochastic column subjected to stochastically distributed loadings using the finite element method," Finite Elements in Analysis and Design, Vol. 11, 1992, pp. 105-115.

11 Ramu, S. A. and Ganesan, R., "Stability of stochastic Leipholz column with stochastic loading," Archive of Applied Mechanics, Vol. 62, 1992a, pp. 363-375.

12 Ramu, S. A. and Ganesan, R., "A Galerkin finite element technique for stochastic field problems," Computer Methods in Applied Mechanics andEngineering, Vol. 105, 1993a, pp. 315-331.

13 Ramu, S. A. and Ganesan, R., "Parametric instability of stochastic columns," International Journal of Solids and Structures, Vol. 30, No. 10, 1993b, pp. 1339-1354.

14 Sankar, T. S., Ramu, S. A., and Ganesan, R., "Stochastic finite element analysis for high speed rotors," Journal of Vibration and Accoustic, ASME, Vol. 115, 1993, pp. 59-64.

15 Adhikari, S., "Complex modes in linear stochastic systems," Proceedings of the First International Conference on Vibration Engineering and Technology of Machinery (VETOMAC-I), edited by K. Venkatraman and C. S. Manohar, Indian Institute of Science, Bangalore, India, October 2000.

16 Adhikari, S., "Complex Modes in Stochastic Systems," Advances in Vibration Engineering, Vol. 3, No. 1, 2004, pp. 1-11.

17 Johnson, N. L. and Kotz, S., Distributions in Statistics: Continuous Univariate Distributions - 2, The Houghton Mifflin Series in Statistics, Houghton Mifflin Company, Boston, USA, 1970.

18 Mathai, A. M. and Provost, S. B., Quadratic Forms in Random Variables: Theory and Applications, Marcel Dekker, Inc., 270 Madison Avenue, New York, NY 10016, USA, 1992.

19 Adhikari, S. and Langley, R. S., "Distribution of eigenvalues of linear stochastic systems," Proceedings of the ninth International Conference on Applications of Statistics and Probability in Civil Engineering (ICASP 9), San Fransisco, California, USA, edited by A. Der-Kiureghian, S. Madanat, and J. M. Pestana, Vol. 1 of Applications of Statistics and Probability in Civil Engineering, Millpress, Rotterdam, Netherlands, July 2003, pp. 201-207.

20 Bleistein, N. and Handelsman, R. A., Asymptotic Expansions of Integrals, Holt, Rinehart and Winston, New York, USA, 1994.

21 Wong, R., Asymptotic Approximations of Integrals, Society of Industrial and Applied Mathematics, Philadelphia, PA, USA, 2001, First published by Academic Press Inc. in 1989.

22 Papadimitriou, C., Beck, J. L., and Katafygiotis, L. S., "Asymptotic expansions for reliability and moments of uncertain systems," Journal of Engineering Mechanics, ASCE, Vol. 123, No. 12, December 1997, pp. 1219-1229.

23 Kapur, J. N. and Kesavan, H. K., Entropy Optimization Principles With Applications, Academic Press Inc., San Diego, CA 92101-4311, 1992.

24 Pearson, E. S., "Note on an approximation to the distribution of non-central $\chi^{2}$," Biometrica, Vol. 46, 1959, pp. 364.

25 Friswell, M. I., "The derivatives of repeated eigenvalues and their associated eigenvectors," ASME Journal of Vibration and Acoustics, Vol. 18, July 1996, pp. 390-397.

26 Fox, R. L. and Kapoor, M. P., "Rates of Change of Eigenvalues and Eigenvectors," AIAA Journal, Vol. 6, No. 12, December 1968, pp. 24262429.

27 Plaut, R. H. and Huseyin, K., "Derivative of eigenvalues and eigenvectors in non-self adjoint systems," AIAA Journal, Vol. 11, No. 2, February 1973, pp. 250-251. 